PROCEEDINGS OF THE

AMERICAN MATHEMATICAL SOCIETY

Volume 135, Number 7, July 2007, Pages 2121-2126

S 0002-9939(07)08715-1

Article electronically published on February 6, 2007

\title{
MULTIPLE SOLUTIONS FOR ELLIPTIC PROBLEMS WITH SINGULAR AND SUBLINEAR POTENTIALS
}

\author{
ALEXANDRU KRISTÁLY AND CSABA VARGA
}

(Communicated by David S. Tartakoff)

\begin{abstract}
For certain positive numbers $\mu$ and $\lambda$, we establish the multiplicity of solutions to the problem

$$
\begin{cases}-\triangle u=\mu \frac{u}{|x|^{2}}+\lambda f(u) & \text { a.e. in } \Omega \\ u=0 & \text { on } \partial \Omega\end{cases}
$$

where $\Omega$ is a bounded open domain in $\mathbb{R}^{N}(N \geq 3)$ containing the origin with smooth boundary $\partial \Omega$, while $f: \mathbb{R} \rightarrow \mathbb{R}$ is continuous, superlinear at zero and sublinear at infinity.
\end{abstract}

\section{INTRODUCTION}

Consider the problem

$$
\left(1_{\mu, \lambda}\right) \quad \begin{cases}-\triangle u=\mu \frac{u}{|x|^{2}}+g(\lambda, u) & \text { in } \Omega, \\ u=0 & \text { on } \partial \Omega,\end{cases}
$$

where $\Omega \subset \mathbb{R}^{N}(N \geq 3)$ is a bounded open domain with smooth boundary $\partial \Omega$ and $0 \in \Omega, g:(0,+\infty) \times \mathbb{R} \rightarrow \mathbb{R}$ is a continuous function and $0 \leq \mu<\bar{\mu}=(N-2)^{2} / 4$.

Problem $\left(1_{0, \lambda}\right)$ has been extensively studied during the last few years (see [7, 8, 10] and references therein), where $g(\lambda, s)=\lambda f(s), f: \mathbb{R} \rightarrow \mathbb{R}$ being a continuous function provided with certain growth properties at zero and infinity, respectively.

When $\mu \neq 0$, problem $\left(1_{\mu, \lambda}\right)$ becomes more delicate due to the presence of the singular potential. Several papers were devoted in order to handle this problem (see [5, 6, 9, 13). For instance, Ferrero and Gazzola [5], and Ruiz and Willem [13], considered $g(\lambda, s)=|s|^{2^{*}-2} s+\lambda s$, establishing for certain values of $\mu$ and $\lambda$ the existence of one nontrivial positive solution for $\left(1_{\mu, \lambda}\right)$. In [9], Montefusco considered $g(\lambda, s)=|s|^{q-2} s$ with either $q \in(1,2)$, or $q \in\left(2,2^{*}\right)$, guaranteeing in both cases a nontrivial solution for $\left(1_{\mu, \lambda}\right)$ whenever $\mu \in(0, \bar{\mu})$ is arbitrarily fixed. Faraci and Livrea [4] exploited Montefusco's result, establishing certain bifurcation theorems which involve the $p$-Laplacian and its corresponding singular term. Very recently, Chen [2, 3] characterized the exact growth order near the origin of the positive solutions of $\left(1_{\mu, \lambda}\right)$ in the case when $g(\lambda, s)=s_{+}^{2^{*}-1}+\lambda s_{+}^{q}(0<q<1)$. By means of this construction, Chen was able to obtain multiple solutions of $\left(1_{\mu, \lambda}\right)$ for certain values of $\lambda>0$ whenever $0 \leq \mu<\bar{\mu}-1(N \geq 5)$.

Received by the editors November 29, 2005 and, in revised form, March 15, 2006.

2000 Mathematics Subject Classification. Primary 35J60, 35J65.

Key words and phrases. Singular potential, sublinearity at infinity, multiple solutions.

(C)2007 American Mathematical Society 
The purpose of this paper is to obtain multiple solutions for the problem

$\left(\mathrm{P}_{\mu, \lambda}\right) \quad \begin{cases}-\triangle u=\mu \frac{u}{|x|^{2}}+\lambda f(u) & \text { a.e. in } \Omega, \\ u=0 & \text { on } \partial \Omega\end{cases}$

(which is nothing but $\left(1_{\mu, \lambda}\right)$ with $g(\lambda, s)=\lambda f(s)$ ) when $f: \mathbb{R} \rightarrow \mathbb{R}$ is superlinear at zero, i.e.

$$
\lim _{s \rightarrow 0} \frac{f(s)}{s}=0
$$

and sublinear at infinity, i.e.

$$
\lim _{|s| \rightarrow+\infty} \frac{f(s)}{s}=0 .
$$

Denoting by $F(s)=\int_{0}^{s} f(t) d t$, we finally assume that

$$
\sup _{s \in \mathbb{R}} F(s)>0 .
$$

Our main result is

Theorem 1. Let $f: \mathbb{R} \rightarrow \mathbb{R}$ be a continuous function which satisfies (f1), (f2) and (f3). Then for every $\mu \in[0, \bar{\mu})$ there exist an open interval $\Lambda_{\mu} \subset(0,+\infty)$ and a real number $\sigma_{\mu}>0$ such that for every $\lambda \in \Lambda_{\mu}$ problem $\left(\mathrm{P}_{\mu, \lambda}\right)$ has at least two distinct, nontrivial weak solutions in $H_{0}^{1}(\Omega)$ whose $H_{0}^{1}$-norms are less than $\sigma_{\mu}$.

We emphasize that hypotheses (f3) cannot be omitted. Indeed, if for instance $f \equiv 0$, then (f1) and (f2) clearly hold, but problem $\left(\mathrm{P}_{\mu, \lambda}\right)$ has only the trivial solution.

Theorem 1 will be proved by means of a recent three critical point result of Bonanno [1] which is actually a refinement of a general principle of Ricceri [1], 12. Furthermore, in Section 3 we will give additional information as far as the localization of the interval $\Lambda_{\mu}$ is concerned (see Remark 2).

\section{Preliminaries}

Let $\Omega$ be a bounded open domain in $\mathbb{R}^{N}(N \geq 3)$ containing the origin with smooth boundary $\partial \Omega$. The space $H_{0}^{1}(\Omega)$ will be endowed by the standard inner product

$$
\langle u, v\rangle_{H_{0}^{1}}=\int_{\Omega} \nabla u \nabla v d x, \quad u, v \in H_{0}^{1}(\Omega),
$$

and by its corresponding norm $\|\cdot\|_{H_{0}^{1}}$. The norm of the dual of $H_{0}^{1}(\Omega)$ will be denoted by $\|\cdot\|_{H^{-1}}$. We recall Hardy's inequality which states that

$$
\int_{\Omega} \frac{u^{2}(x)}{|x|^{2}} d x \leq \frac{1}{\bar{\mu}}\|u\|_{H_{0}^{1}}^{2}, \quad u \in H_{0}^{1}(\Omega),
$$

where $\bar{\mu}=(N-2)^{2} / 4$ (see [6]).

The usual norm on $L^{p}(\Omega)$ will be denoted by $\|\cdot\|_{L^{p}}$. The Sobolev embedding constant of the compact embedding $H_{0}^{1}(\Omega) \hookrightarrow L^{p}(\Omega), p \in\left[1,2^{*}\right)$, will be denoted by $c_{p}>0$, i.e. $\|u\|_{L^{p}} \leq c_{p}\|u\|_{H_{0}^{1}}$, for every $u \in H_{0}^{1}(\Omega)$.

Let $f: \mathbb{R} \rightarrow \mathbb{R}$ be a continuous function and $F(s)=\int_{0}^{s} f(t) d t$. We introduce the energy functional $\mathcal{E}_{\mu, \lambda}: H_{0}^{1}(\Omega) \rightarrow \mathbb{R}$ associated to $\left(\mathrm{P}_{\mu, \lambda}\right)$, i.e.

$$
\mathcal{E}_{\mu, \lambda}(u)=\Phi_{\mu}(u)-\lambda J(u), \quad u \in H_{0}^{1}(\Omega)
$$


where

$$
\Phi_{\mu}(u)=\frac{1}{2}\|u\|_{H_{0}^{1}}^{2}-\frac{\mu}{2} \int_{\Omega} \frac{u^{2}(x)}{|x|^{2}} d x \quad \text { and } \quad J(u)=\int_{\Omega} F(u(x)) d x, \quad u \in H_{0}^{1}(\Omega) .
$$

As long as (f2) is verified, a standard argument shows that $\mathcal{E}_{\mu, \lambda}: H_{0}^{1}(\Omega) \rightarrow \mathbb{R}$ is of class $C^{1}$ and its critical points are exactly the weak solutions of $\left(\mathrm{P}_{\mu, \lambda}\right)$.

Therefore, it is enough to show the existence of multiple critical points of $\mathcal{E}_{\mu, \lambda}$ : $H_{0}^{1}(\Omega) \rightarrow \mathbb{R}$ for the parameters $\mu$ and $\lambda$ specified in Theorem 1 , This fact will be carried out by means of the following recent critical point result.

Theorem 2 ([1, Theorem 2.1]). Let $X$ be a separable and reflexive real Banach space, and let $\Phi, J: X \rightarrow \mathbb{R}$ be two continuously Gâteaux differentiable functionals. Assume that there exists $x_{0} \in X$ such that $\Phi\left(x_{0}\right)=J\left(x_{0}\right)=0$ and $\Phi(x) \geq 0$ for every $x \in X$ and that there exists $x_{1} \in X, \rho>0$ such that

(i) $\rho<\Phi\left(x_{1}\right)$;

(ii) $\sup _{\Phi(x)<\rho} J(x)<\rho \frac{J\left(x_{1}\right)}{\Phi\left(x_{1}\right)}$.

Further, put

$$
\bar{a}=\frac{\zeta \rho}{\rho \frac{J\left(x_{1}\right)}{\Phi\left(x_{1}\right)}-\sup _{\Phi(x)<\rho} J(x)},
$$

with $\zeta>1$, assume that the functional $\Phi-\lambda J$ is sequentially weakly lower semicontinuous and satisfies the Palais-Smale condition, and

(iii) $\lim _{\|x\| \rightarrow+\infty}(\Phi(x)-\lambda J(x))=+\infty$, for every $\lambda \in[0, \bar{a}]$.

Then there is an open interval $\Lambda \subseteq[0, \bar{a}]$ and a number $\sigma>0$ such that for each $\lambda \in \Lambda$, the equation $\Phi^{\prime}(x)-\lambda J^{\prime}(x)=0$ admits at least three solutions in $X$ having norm less than $\sigma$.

\section{Proof of Theorem 1}

Through this section, we suppose that the assumptions of Theorem 1 are fulfilled. In order to conclude the proof, we apply Theorem 2 by choosing $X=H_{0}^{1}(\Omega)$ as well as $\Phi=\Phi_{\mu}$ and $J$ as in (2). Due to (1) we have at once that $\Phi_{\mu}(u) \geq 0$ for every $\mu \in[0, \bar{\mu})$ and $u \in X$.

Lemma 1. For every $\mu \in[0, \bar{\mu}]$ and $\lambda \in \mathbb{R}$, the functional $\mathcal{E}_{\mu, \lambda}$ is sequentially weakly lower semicontinuous on $H_{0}^{1}(\Omega)$.

Proof. Due to (f2), there exists $c>0$ such that

$$
|f(s)| \leq c(1+|s|), s \in \mathbb{R} .
$$

Thus, the sequentially weak continuity of $J$ is achieved in a standard way, by means of the compact embedding $H_{0}^{1}(\Omega) \hookrightarrow L^{2}(\Omega)$. On the other hand, using the concentration-compactness principle, Montefusco proved in [9, Theorem 3.2] the sequentially weakly lower semicontinuity of $\Phi_{\mu}$ for every $\mu \in[0, \bar{\mu}]$.

Lemma 2. For every $\mu \in[0, \bar{\mu})$ and $\lambda \in \mathbb{R}$, the functional $\mathcal{E}_{\mu, \lambda}$ is coercive and satisfies the Palais-Smale condition. 
Proof. Let us fix $\mu \in[0, \bar{\mu})$ and $\lambda \in \mathbb{R}$ arbitrarily. By (f2), there exists $\delta=\delta(\mu, \lambda)>$ 0 such that for every $|s|>\delta$ one has

$$
|f(s)|<\frac{1}{2}(1-\mu / \bar{\mu}) c_{2}^{-2}(1+|\lambda|)^{-1}|s| .
$$

After integration, we obtain

$$
|F(s)| \leq \frac{1}{2}(1-\mu / \bar{\mu}) c_{2}^{-2}(1+|\lambda|)^{-1}|s|^{2}+\max _{|t| \leq \delta}|f(t)||s| \quad \text { for all } s \in \mathbb{R} .
$$

Thus, for every $u \in H_{0}^{1}(\Omega)$ we have

$$
\begin{aligned}
\mathcal{E}_{\mu, \lambda}(u) & \geq \frac{1}{2}(1-\mu / \bar{\mu})\|u\|_{H_{0}^{1}}^{2}-|\lambda| \int_{\Omega}|F(u(x))| d x \\
& \geq \frac{1}{2}(1-\mu / \bar{\mu})(1+|\lambda|)^{-1}\|u\|_{H_{0}^{1}}^{2}-c_{1}|\lambda| \max _{|t| \leq \delta}|f(t)|\|u\|_{H_{0}^{1}} .
\end{aligned}
$$

If $\|u\|_{H_{0}^{1}} \rightarrow+\infty$ we conclude that $\mathcal{E}_{\mu, \lambda}(u) \rightarrow+\infty$ as well, i.e. $\mathcal{E}_{\mu, \lambda}$ is coercive.

Now, let $\left\{u_{n}\right\}$ be a sequence in $H_{0}^{1}(\Omega)$ such that $\left\{\mathcal{E}_{\mu, \lambda}\left(u_{n}\right)\right\}$ is bounded and $\left\|\mathcal{E}_{\mu, \lambda}^{\prime}\left(u_{n}\right)\right\|_{H^{-1}} \rightarrow 0$. Since $\mathcal{E}_{\mu, \lambda}$ is coercive, the sequence $\left\{u_{n}\right\}$ is bounded. Up to a subsequence, we may suppose that $u_{n} \rightarrow u$ weakly in $H_{0}^{1}(\Omega)$, and $u_{n} \rightarrow u$ strongly in $L^{2}(\Omega)$ for some $u \in H_{0}^{1}(\Omega)$. On the other hand, we have

$$
\begin{aligned}
(1-\mu / \bar{\mu})\left\|u_{n}-u\right\|_{H_{0}^{1}}^{2} \leq & \left\|u_{n}-u\right\|_{H_{0}^{1}}^{2}-\mu \int_{\Omega} \frac{\left(u_{n}(x)-u(x)\right)^{2}}{|x|^{2}} d x \\
= & \mathcal{E}_{\mu, \lambda}^{\prime}\left(u_{n}\right)\left(u_{n}-u\right)+\mathcal{E}_{\mu, \lambda}^{\prime}(u)\left(u-u_{n}\right) \\
& +\lambda \int_{\Omega}\left[f\left(u_{n}(x)\right)-f(u(x))\right]\left(u_{n}(x)-u(x)\right) d x .
\end{aligned}
$$

It is clear the first two terms from the last expression tend to 0 , while by means of (3) one has

$$
\begin{gathered}
\int_{\Omega}\left|f\left(u_{n}(x)\right)-f(u(x)) \| u_{n}(x)-u(x)\right| d x \\
\leq c\left[2(\operatorname{meas} \Omega)^{1 / 2}+\left\|u_{n}\right\|_{L^{2}}+\|u\|_{L^{2}}\right]\left\|u_{n}-u\right\|_{L^{2}} \rightarrow 0
\end{gathered}
$$

as $n \rightarrow \infty$. Thus we proved that $\left\|u_{n}-u\right\|_{H_{0}^{1}} \rightarrow 0$.

Lemma 3. For every $\mu \in[0, \bar{\mu})$,

$$
\lim _{\rho \rightarrow 0^{+}} \frac{\sup \left\{J(u): \Phi_{\mu}(u)<\rho\right\}}{\rho}=0 .
$$

Proof. Fix $\mu \in[0, \bar{\mu})$. Due to (f1), for an arbitrarily small $\varepsilon>0$ there exists $\delta=\delta(\varepsilon)$ such that

$$
|f(s)|<\frac{\varepsilon}{4}(1-\mu / \bar{\mu}) c_{2}^{-2}|s| \text { for all }|s|<\delta .
$$

For a fixed $p \in\left(2,2^{*}\right)$, combining (3) with the above inequality, one has

$$
|F(s)| \leq \frac{\varepsilon}{4}(1-\mu / \bar{\mu}) c_{2}^{-2}|s|^{2}+c(1+\delta) \delta^{1-p}|s|^{p} \quad \text { for all } s \in \mathbb{R} .
$$

For $\rho>0$ define the sets

$$
S_{\rho}^{1}=\left\{u \in H_{0}^{1}(\Omega): \Phi_{\mu}(u)<\rho\right\} ; \quad S_{\rho}^{2}=\left\{u \in H_{0}^{1}(\Omega):(1-\mu / \bar{\mu})\|u\|_{H_{0}^{1}}^{2}<2 \rho\right\} .
$$


Thanks to (1), $S_{\rho}^{1} \subseteq S_{\rho}^{2}$. Moreover, by using (4), for every $u \in S_{\rho}^{2}$ we have

$$
J(u) \leq \frac{\varepsilon}{2} \rho+c(1+\delta) \delta^{1-p} c_{p}^{p} 2^{p / 2}(1-\mu / \bar{\mu})^{-p / 2} \rho^{p / 2} \equiv \frac{\varepsilon}{2} \rho+c^{\prime} \rho^{p / 2} .
$$

Thus there exists $\rho(\varepsilon)>0$ such that for every $0<\rho<\rho(\varepsilon)$

$$
0 \leq \frac{\sup _{u \in S_{\rho}^{1}} J(u)}{\rho} \leq \frac{\sup _{u \in S_{\rho}^{2}} J(u)}{\rho} \leq \frac{\varepsilon}{2}+c^{\prime} \rho^{\frac{p-2}{2}}<\varepsilon
$$

which completes the proof.

Let $s_{0} \in \mathbb{R}$ such that $F\left(s_{0}\right)>0$; see (f3). Also choose $R_{0}>0$ in such a way that $R_{0}<\operatorname{dist}(0, \partial \Omega)$. For $\sigma \in(0,1)$ define

$$
u_{\sigma}(x)=\left\{\begin{array}{lll}
0, & \text { if } \quad x \in \mathbb{R}^{N} \backslash B_{N}\left(0, R_{0}\right), \\
s_{0}, & \text { if } \quad x \in B_{N}\left(0, \sigma R_{0}\right), \\
\frac{s_{0}}{R_{0}(1-\sigma)}\left(R_{0}-|x|\right), & \text { if } \quad x \in B_{N}\left(0, R_{0}\right) \backslash B_{N}\left(0, \sigma R_{0}\right),
\end{array}\right.
$$

where $B_{N}(0, r)$ denotes the $N$-dimensional open ball with center 0 and radius $r>0$. It is clear that $u_{\sigma} \in H_{0}^{1}(\Omega)$. Moreover, denoting by $\omega_{N}$ the volume of the $N$ dimensional unit ball, one has

$$
\left\|u_{\sigma}\right\|_{H_{0}^{1}}^{2}=s_{0}^{2}(1-\sigma)^{-2}\left(1-\sigma^{N}\right) \omega_{N} R_{0}^{N-2}
$$

and

$$
J\left(u_{\sigma}\right) \geq\left[F\left(s_{0}\right) \sigma^{N}-\max _{|t| \leq\left|s_{0}\right|}|F(t)|\left(1-\sigma^{N}\right)\right] \omega_{N} R_{0}^{N} .
$$

For $\sigma$ close enough to 1, the right-hand side of the last inequality becomes strictly positive; choose such a number, say $\sigma_{0}$.

Proof of Theorem 1 completed. Fix $\mu \in[0, \bar{\mu})$. Due to Lemma 3 , we may choose $\rho_{0}>0$ such that

$$
\begin{gathered}
2 \rho_{0}<(1-\mu / \bar{\mu})\left\|u_{\sigma_{0}}\right\|_{H_{0}^{1}}^{2}, \\
\frac{\sup \left\{J(u): \Phi_{\mu}(u)<\rho_{0}\right\}}{\rho_{0}}<\frac{2 ;\left[F\left(s_{0}\right) \sigma_{0}^{N}-\max _{|t| \leq\left|s_{0}\right|}|F(t)|\left(1-\sigma_{0}^{N}\right)\right] \omega_{N} R_{0}^{N}}{\left\|u_{\sigma_{0}}\right\|_{H_{0}^{1}}^{2}} .
\end{gathered}
$$

By choosing $x_{1}=u_{\sigma_{0}}$, hypotheses (i) and (ii) of Theorem 2 are verified. Define

$$
\bar{a}=\bar{a}_{\mu}=\frac{1+\rho_{0}}{\frac{J\left(u_{\sigma_{0}}\right)}{\Phi_{\mu}\left(u_{\sigma_{0}}\right)}-\frac{\sup \left\{J(u): \Phi_{\mu}(u)<\rho_{0}\right\}}{\rho_{0}}} .
$$

Taking into account Lemmas 1 and 2, and putting $x_{0}=0$, all the assumptions of Theorem 2 are verified. Thus there exist an open interval $\Lambda_{\mu} \subset\left[0, \bar{a}_{\mu}\right]$ and a number $\sigma_{\mu}>0$ such that for each $\lambda \in \Lambda_{\mu}$, the equation $\mathcal{E}_{\mu, \lambda}^{\prime}(u) \equiv \Phi_{\mu}^{\prime}(u)-\lambda J^{\prime}(u)=0$ admits at least three solutions in $H_{0}^{1}(\Omega)$ having $H_{0}^{1}$-norm less than $\sigma_{\mu}$. Since one of them may be the trivial one $(f(0)=0$, see (f1)), we still have at least two distinct, nontrivial solutions of $\left(\mathrm{P}_{\mu, \lambda}\right)$ with the required properties.

Remark 1. Since $f(0)=0$, one can consider the continuous function $s \mapsto f\left(s_{+}\right)$ instead of $f$, obtaining nonpositive solutions of the problem $\left(\mathrm{P}_{\mu, \lambda}\right)$. Here, $s_{+}=$ $\max \{s, 0\}$. Moreover, if $f$ is locally Lipschitz, the solutions belong to $C^{2}(\Omega \backslash\{0\})$. 
Remark 2. It is important to know explicit estimations of the intervals $\Lambda_{\mu}, \mu \in$ $[0, \bar{\mu})$, guaranteed by Theorem 1 . In order to give such an estimation, let us fix $s_{0}$, $R_{0}$, and $\sigma_{0}$ as in Section 3 . Let $\mu \in[0, \bar{\mu})$. Based on Lemma 3 , one can assume that $\rho_{0}<1$ and

$$
\frac{\sup \left\{J(u): \Phi_{\mu}(u)<\rho_{0}\right\}}{\rho_{0}}<\frac{J\left(u_{\sigma_{0}}\right)}{2 \Phi_{\mu}\left(u_{\sigma_{0}}\right)} .
$$

Thus, according to (5), one has $\bar{a}_{\mu}<\frac{4 \Phi_{\mu}\left(u_{\sigma_{0}}\right)}{J\left(u_{\sigma_{0}}\right)}$. In conclusion, we have

$$
\Lambda_{\mu} \subset\left[0,2\left(1-\frac{\mu}{\bar{\mu}}\right)\left(\frac{s_{0}}{R_{0}}\right)^{2} \frac{\left(1-\sigma_{0}\right)^{-2}\left(1-\sigma_{0}^{N}\right)}{F\left(s_{0}\right) \sigma_{0}^{N}-\max _{|t| \leq\left|s_{0}\right|}|F(t)|\left(1-\sigma_{0}^{N}\right)}\right] .
$$

\section{ACKNOWLEDGMENTS}

A. Kristály has been supported by a Domus Hungarica Fellowship at the University of Debrecen where the main part of this paper has been written. He is grateful to Professor Biagio Ricceri for valuable remarks during the preparation of the manuscript. Both authors were supported in part by the Research Center of Sapientia Foundation.

\section{REFERENCES}

[1] G. Bonanno, Some remarks on a three critical points theorem, Nonlinear Analysis TMA, 54 (2003), 651-665. MR1983441 (2004d:49010)

[2] J. Chen, Exact local behavior of positive solutions for a semilinear elliptic equation with Hardy term, Proc. Amer. Math. Soc., 132 (2004), no. 11, 3225-3229. MR2073296 (2006f:35079)

[3] J. Chen, Multiple positive solutions for a class of nonlinear elliptic equations, J. Math. Anal. Appl., 295 (2004), no. 2, 341-354. MR2072017 (2005c:35084)

[4] F. Faraci, R. Livrea, Bifurcation theorems for nonlinear problems with lack of compactness, Ann. Polon. Math., 82.1 (2003), 77-85. MR2041400 (2005a:35098)

[5] A. Ferrero, F. Gazzola, Existence of solutions for singular critical growth semilinear elliptic equations, J. Differential Equations, 177 (2001), 494-522. MR.1876652 (2002m:35068)

[6] J. P. Garcia Azorero, I. Peral Alonso, Hardy inequalities and some critical elliptic and parabolic problems, J. Differential Equations, 144 (1998), 441-476. MR1616905 (99f:35099)

[7] Z. Guo, J. R. L. Webb, Large and small solutions of a class of quasilinear elliptic eigenvalue problems, J. Differential Equations, 180 (2002), 1-50. MR.1890596 (2002k:35246)

[8] D. D. Hai, On a class of sublinear quasilinear elliptic problems, Proc. Amer. Math. Soc., 131 (2003), 2409-2414. MR1974638 (2003m:35090)

[9] E. Montefusco, Lower semicontinuity of functionals via concentration-compactness principle, J. Math. Anal. Appl., 263 (2001), 264-276. MR1865280(2002h:35053)

[10] J. Saint Raymond, On the multiplicity of solutions of the equation $-\triangle u=\lambda \cdot f(u), \quad J$. Differential Equations, 180 (2002), 65-88. MR1890598 (2003a:35141)

[11] B. Ricceri, On a three critical points theorem, Arch. Math. (Basel) 75 (2000), 220-226. MR:1780585 (2001h:49012)

[12] B. Ricceri, Existence of three solutions for a class of elliptic eigenvalue problems, Math. Comput. Modelling, 32 (2000), 1485-1494. MR.1800671 (2001j:35220)

[13] D. Ruiz, M. Willem, Elliptic problems with critical exponents and Hardy potential, J. Differential Equations, 190 (2003), 524-538. MR1970040 (2004c:35138)

University of Babeş-Bolyai, Faculty of Economics, Str. Teodor Mihali 58-60, RO400591, Cluj Napoca, Romania

E-mail address: alexandrukristaly@yahoo.com

University of Babeş-Bolyai, Faculty of Mathematics and Computer Science, Str. Kogalniceanu 1, RO-400084, Cluj-Napoca, Romania

E-mail address: csvarga@math.ubbcluj.ro 